\title{
Periodic Changes of the Accretion Geometry in the Nearly-Synchronous Polar RX J1940.1-1025
}

\author{
Ralf D. Geckeler and Rüdiger Staubert \\ Institut für Astronomie und Astrophysik, Astronomie, Universität \\ Tübingen, Waldhäuserstr. 64, D-72076 Tübingen, Germany
}

\begin{abstract}
RX J1940.1-1025 is a newly discovered magnetic Cataclysmic Variable and one of only three known polars with a slightly non-synchronous $(<3 \%)$ rotation of the white dwarf primary. The accretion geometry between the infalling accretion stream and the magnetic field of the WD thus changes periodically with the beat period of $50.0 \mathrm{~d}$ between the orbital and the spin period of the WD. The accretion thus occurs along different field lines, depending on the beat phase.

For the first time we have observed this effect as a periodic shift in the timings of the absorption troughs in the optical and X-ray pulse profiles of RX J1940.1-1025 with phase of the beat period. There is evidence for a secular decrease of the spin period of RX J1940.1-1025 with a synchronization time scale of the order of $10^{2} \mathrm{y}$.
\end{abstract}

\section{Observational Data}

Through observations with ROSAT, RX J1940 has been identified as the source of the periodically modulated X-ray flux (Madejski et al. 1993, Staubert et al. 1994) which had previously been associated with the Active Galaxy NGC 6814. Fig. 1(left) shows the X-ray (ROSAT PSPC 0.1-2.4keV) and optical (our photometry) pulse profiles of RX J1940. The data have been corrected for the shifts according to the best fit dipole model and the quadratic component of the spin ephemeris and then folded with the WD spin period $P_{\text {spin }}=12150.4 \mathrm{~s}$. The double peak structure of the X-ray pulse profile can be explained by an X-ray bright phase with a strong absorption by the accretion funnel directly above the WD surface cutting into the intensity peak, when the observer looks nearly parallel to the field lines above the accretion region $(\Phi=1.0)$. This absorption trough is prominent in the optical pulse profile as a broader and shallower trough feature, too. We have extracted the timings of the troughs from our extended optical and ROSAT X-ray data sets, including the photometry from Patterson et al. (1995) and Watson et al. (1995).

\section{Results}

The timings of the absorption troughs have been analyzed by simultaneously fitting a linear and quadratic spin ephemeris plus a modelling of the accretion process, where the infalling matter is captured in a centered dipole field geom- 

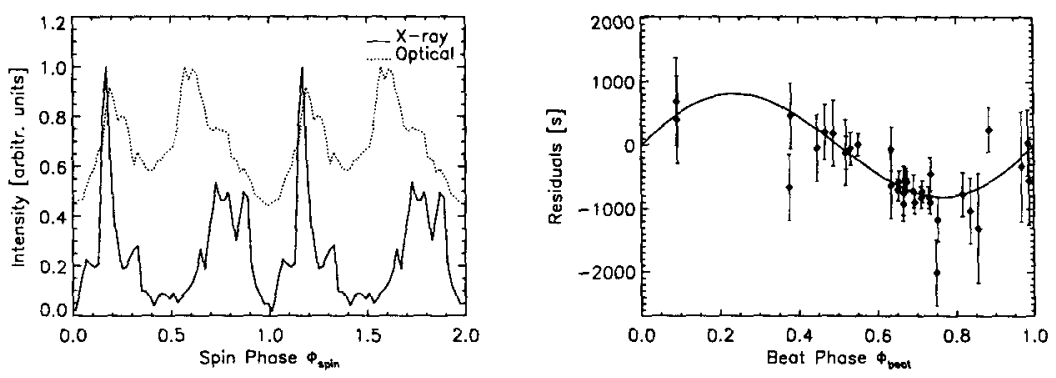

Figure 1. Left: Optical and X-ray pulse profiles of RX J1940, folded with the WD spin period of $12150.4 \mathrm{~s}$ (see text).

Right: Residuals of the absorption troughs (optical plus X-ray data) in RX J1940 from the linear plus quadratic spin ephemeris as a function of beat phase $\Phi_{\text {beat }}$, and the best fit dipole model.

etry and accreted along different field lines during the beat period (Geckeler et al. 1996). Fig. 1(right) shows the resulting residuals of the troughs (optical and X-ray data from 1993-96) from the linear plus quadratic spin ephemeris, folded with the beat period $P_{\text {beat }}$. The dipole accretion model provides a valid description of the data over the three year time span. The data refine the WD spin period $P_{\text {spin }}=12150.40 \pm 0.08 \mathrm{~s}$ with the zero point (center of trough) at HJD $2449549.7359 \pm 0.0042$. There is evidence for a $\dot{P}_{\text {spin }}$ of the order of $(-1 \pm 0.3) \cdot 10^{-8} \mathrm{~s} / \mathrm{s}$, with a resulting synchronization time scale of $10^{2} \mathrm{y}$, comparable to the $170 \mathrm{y}$ seen in V1500 Cyg. The data allow to give a tight constraint only on the combined parameter $\sin \beta \sqrt{d / R_{w d}}=3.60 \pm 0.17$ (for $\beta>40^{\circ}$ ), with the colatitute $\beta$ and the mean distance $\mathrm{d}$ from the WD, at which the infalling matter is captured by the field. The fit results in $d>7 R_{w d}$ at the $68 \%$ confidence level, leading to $B_{p o l}>10^{7} \mathrm{G}$, as expected for a polar.

Acknowledgments. This work was supported by DARA under grant 50 OR 96031 and by DFG under grant ST 173/22-1. S. Friedrich, G.Lamer and M. König contributed optical observations used in this work.

\section{References}

Friedrich, S., Staubert, R., Lamer, G. et al. 1996, A\&A, 306, 860

Geckeler, R.D. and Staubert, R., et al. 1996, A\&A, in prep.

Madejski, G.M., Done, C., Turner, T.J. et al. 1993, Nat, 365, 626

Patterson, J., Skillman, D.R., Thorstensen, J. et al. 1995, PASP, 107, 307

Staubert, R., König, M., Friedrich, S. et al. 1994, A\&A, 288, 513

Staubert, R., Friedrich, S., Lamer, G. et al. 1995, In: Proc. Cape Workshop on Magnetic CVs, p. 264, Eds. Buckley, D.A.H. and Warner, B., ASP Conf. Series 85

Watson, M.G., Rosen, S.R., O’Donoghue, D. et al. 1995, MNRAS, 273, 681 